International Journal of Pure and Applied Mathematics

Volume 82 No. 5 2013, 719-729

ISSN: 1311-8080 (printed version); ISSN: 1314-3395 (on-line version)

url: http://www.ijpam.eu

doi: http://dx.doi.org/10.12732/ijpam.v82i5.5

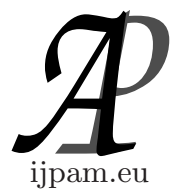

\title{
ODD AND EVEN NUMBER CULTURES
}

\author{
Yutaka Nishiyama \\ Department of Business Information \\ Faculty of Information Management \\ Osaka University of Economics \\ 2, Osumi Higashiyodogawa Osaka, 533-8533, JAPAN
}

\begin{abstract}
Japanese prefer odd numbers, while Westerners prefer even numbers. This is clear from the distribution of number-related words in Japanese and English dictionaries. This paper explains the reason for this cultural difference by surveying the history of numbers, Yin-Yang thought from ancient China, ancient Greek philosophy, and modern European mathematics. The author also mentions that while odd and even are only mathematical concepts, understanding the culture and history of individual countries contributes to world peace.
\end{abstract}

AMS Subject Classification: 01A27, 00A09, 97A40

Key Words: odd number, even number, Japanese and English, history of numbers, Yin-Yang thought

\section{Japanese Prefer Odd Numbers}

Japanese like the number "one." They also like the numbers "three", "five", and "seven.", as in the Seven-Five-Three Festival (Shichigosan Festival), in which 3 -year-old boys and girls, 5-year-old boys, and 7-year-old girls go to shrines to celebrate their growth. It could have been ages six, four, or two, but ages three, five, and seven were it. Similarly, there is a custom where festivals are

Received: October 13, 2012

(c) 2013 Academic Publications, Ltd. url: www.acadpubl.eu 
held on odd numbered days in odd-numbered months. January 1 (first day of the first month) is New Year's Day, March 3 (third day of the third month) the Girl's Festival, May 5 (fifth day of the fifth month) the Boy's Festival, July 7 (seventh day of the seventh month) the Star Festival, and September 9 (ninth day of the ninth month) is the Chrysanthemum Festival (this reinforces the significance of the odd-number nine). Haiku poetry is composed in lines of fiveseven-five syllables; tanka poetry in lines of five-seven-five-seven-seven syllables; Chinese poetry in quatrains with five-character (syllable) and seven-character lines. Cheer groups also clap their hands in three-three-seven beat rhythms.

In contrast, even numbers do not have good associations. "Two" means "to divide" (or "to part, separate"), "four" is associated with death, and "six" as in the phrase "rokudenashi", means "good-for-nothing." At wedding ceremonies, people give gifts of 10, 000 yen, 30, 000 yen, and fifty thousand yen. No-one gives a gift of 20, 000 or 40, 000 yen. Similarly, at funerals the condolence payments are all in odd numbers. This may reflect the influence of Yin-Yang thought from China, in which odd numbers are "Yang" (masculine). Hospital sickrooms and parking lots avoid the number "four" (which is homophonous with "death"). This is simply a matter of homophony and has no scientific grounds. Superstitions related to "four" is perhaps specific to Japan. People like odd numbers and dislike even numbers. However, there are two exceptions: "eight" (hachi) which can be taken as referring to "increasing prosperity" (because the Japanese character for eight is shaped like a fan, which starts narrow and broadens), and "nine" (ku) which can be taken as referring to "suffering", which is also read as "ku".

\section{The Distribution of Numerals}

It is clear that a lot of odd numbers lie embedded and unseen in Japanese culture. Given this factor, I used a PC to conduct a survey of number-related words. In order to visualize the trend, I decided to search for word-initials (initial word-position agreement). In this way, I was able to search out all number-related words from one to nine.

In the case of English, the cardinal numbers from 1 to 9 are first of all one, two, three, four, five, six, seven, eight, and nine, and the ordinal numbers are first, second, third, fourth, fifth, sixth, seventh, eighth, and ninth. In addition, since there were more ordinal numbers, I added the following as data: single, double, and triple corresponding to one, two, and three; once, twice, and thrice corresponding to one, two, and three; half and quarter referring respectively to 
half of one and one quarter of one corresponding to two and four; and "couple" and "pair" referring to two.

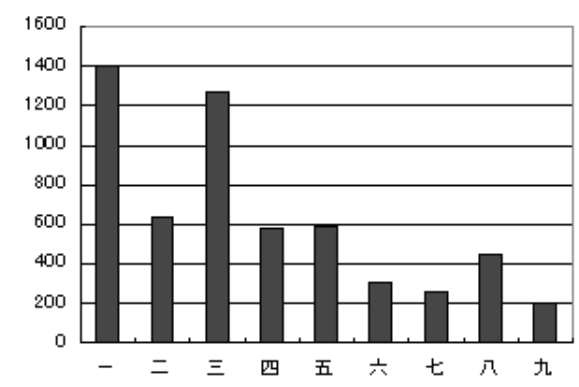

Figure 1: Distribution of Numerals (Japanese)

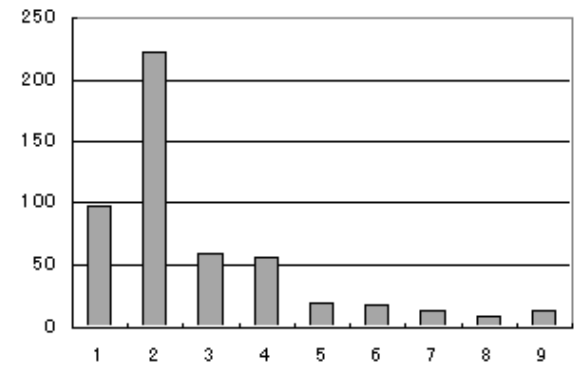

Figure 2: Distribution of Numerals (English)

As for the databases that were used for the acquisition of this data, the electronic version of the second edition of "Daijirin" (Large Dictionary of Japanese) published by (Sanseido Co., Ltd.) was used for the Japanese, and the sixth edition of "New English-Japanese Dictionary" (Kenkyusha) for the English, and the free software supplied by Excite Japan Co., Ltd. (URL: http://www.excite. co.jp/) was used. Although the number of entries for English is slightly smaller than for Japanese; the trend can be clearly seen. (see Figure 1 and 2.)

The graph showing the distribution of numerals reveals the following points: numbers one and three are most common in Japanese, followed by two, four, and five, which are used in approximately equal proportions, followed by six to nine 
in that order. Eight seems to be the most common in the six-to-nine sequence. In the case of English, two is most frequent, followed by one. Next, three and four are used in approximately equal proportions, and numbers five to nine trail without much difference between them. Looking at the distribution from a different angle, the odd numbers one, three, and five seem to predominate in Japanese while the even numbers two and four seem to predominate in English.

With that, it appears that the Japanese language has a cultural setting that favors the odd numbers three and five while English has a cultural setting that favors the even numerals two, four, and six. Language is a means of communication; as such it is a cultural legacy passed on throughout the long history of humanity. The human race is generally divided into white, black, and yellow, and there are thought to be around 5,000 languages in the world. Besides English and Japanese, there must be frequency graphs of word distribution for various other languages.

\section{Westerners who Emphasize Symmetry}

In Japan, there are always discussions about whether a two-party system can be realized. It is thought that politics will improve if a two-party system such as the American Democratic and Republican parties, and the British Labor and Conservative parties can be realized.

Since Meiji, however, Japan has never realized a two-party system. Currently, The Democratic Party of Japan is aiming at a two-party system, but it seems that Japan is not congenial to the two-party system. Is it too bold to suggest that this is because Japanese culture is conducive to odd numbers while American and British culture is conducive to even numbers? For Japan, it is either one or three.

Moreover, a new, two-thousand yen note was issued, but the use of it hasn?ft taken root. One of the causes is thought to be the fact that current vending machines do not support the new two-thousand yen note, but I suspect there may be other causes. America has two-dollar and twenty-dollar notes, and the UK has the two-pound coin and twenty-pound note. Why is this? Doesn't this suggest that there may be some connection with cultures that are conducive to odd numbers and those that are conducive to even numbers?

There is an interesting story about the rainbow. According to a book by Takao Suzuki entitled Nihongo to Gaikokugo ("Japanese and Foreign Languages") published by Iwanami Shinsho, the rainbow is perceived to have seven colors in Japanese culture. He presents a very interesting analysis of why the 
rainbow is perceived as having five or six colors, depending on the country. In Japan, the seven primary colors of the rainbow are 赤(aka), 橙(dai-dai), 黄(ki), 緑(midori), 青(ao), 藍(ai), and 紫(murasaki); these correspond to red, orange, yellow, green, blue, indigo, and purple in English. When the Western cultural zone refers to the rainbow as having only six colors, indigo is omitted. In Japan, the rainbow is commonly perceived as consisting of seven colors while in Europe and America it is commonly perceived as consisting of six colors. However, a rainbow actually consists of a continuous spectrum of colors, so neither perception is correct. Therefore, isn't it possible that people's perception of the rainbow as consisting of either seven or six colors reflects whether their culture favors odd numbers or even numbers?

The "gobosei" (pentagram) and "rokubosei" (hexagram) used in Chinese /Japanese-style fortune-telling represent shapes of stars. The "gobosei," also known as the pentagram, is well known as forming the five-petaled bellflower family crest of Abe Seimei (a noted fortune-teller in Japan). The five points of the "gobosei" (pentagram) are represented by wood, fire, earth, metal, and water in the Yin-Yang -Five elements of thinking. The "rokubosei" (hexagram), which is also the shape of the "star of David,?h the symbol of Judaism, is written with two triangles, and is also regarded as symbolizing the union of Yin (female) and Yang (male). Although stars are actually spherical in shape, they are expressed by the shapes of the pentagon and hexagon. Hence, the "gobosei" (pentagram) can also be regarded as reflecting a worship of odd numbers while the "rokugosei" (hexagram) may be regarded as reflecting a worship of even numbers.

Next I will cite examples of the way in which numbers are perceived differently depending on the culture of the country. There is a Japanese saying that goes "gojuppo hyakupo" (50 steps or 100 steps). The "Modern EnglishJapanese dictionary" (Kenkyusha, 1973) translates this saying as "It's six of one and half-a-dozen of the other." When there is little difference between two things, this is referred to as "50 or 100" in Japanese and "six or a half-dozen" in English. The duodecimal and sexagesimal systems spread in the West, and these are rational numbering systems with many divisors. Is the history of odd and even numbers in the West and the East also reflected in proverbs?

There is a proverb that goes "sanninyoreba monjunochie" (the counsel of three persons will result in wisdom like that of Monju, the Bodhisattva of Wisdom). These proverbs purports that even three fools can come together and have discussions that will result in wisdom like that of Monju, the Bodhisattva of Wisdom. According to the "New English-Japanese Dictionary" (Kenkyusha, 1995), the corresponding English proverb is "Two heads are better than one." 
In Japanese, three are better than two, while in English two are better than one. Doesn't this perhaps show the difference between favoring odd numbers (three people) and favoring even numbers (two people)?

\section{Odd and Even Numbers in Various Languages}

What happens to odd and even numbers in other languages? In ancient China, the characters奇 (qi or ki) and Char1 (ou or gu) were used to express odd and even; however, nowadays奇 (qi or ki) and偶 (ou or gu) are used. The character Char1 means "ploughshare" (two persons lined up to plough together. Similarly, singular numbers are sometimes used to refer to odd numbers and dual numbers, as even numbers. Korean Hangul writing uses Char2 to refer to the odd number and Char3, to the even number. In Indonesian, the term "ganjil" refers to the odd number and the term "genap", to the even number. Like so, China has had a great influence in the East.

\section{Char1: 耦 Char2: 기수 Char3: 우수}

What about the West? English uses the terms "even number" and "odd number." In Spanish, the terms used are número par (even number) and número impar (odd number). In French, the terms used are nombre pair (even number) and nombre impair (odd number). In Italian, the terms used are nùmero pari (even number) and nùmero dispari (odd number). In German, the terms used are gerade Zahl (even numbers) and ungerade Zahl (odd numbers). In European languages, the word referring to the even number is the basic term and the term referring to the odd number is the negated form of the basic term. In old English, the term for the odd number was also referred to as the "uneven number," following the same principle. In current English, a completely different term, the "odd number" is used.

By now, one wonders why Japanese prefer odd numbers. What could be the reason for liking numbers known as "odd," which means eccentric, a crackpot, and a bizarre person? A preference for odd numbers is in itself, strange.

Let's look at words that use the kanji 奇 (qi or ki). There are words with positive connotations, such as 奇才 (kisai “a prodigy"), 奇抜 (kibatsu "unique"), 奇特 (kitoku "praiseworthy"), and 奇跡的 (kisekiteki "miraculous"). There are also words with negative connotations, such as 奇怪 (kikai "mysterious"), 奇 行 (kiko "eccentric conduct"), 奇声 (kisei "strange voice"), and 奇人 (kijin “an eccentric person"). The ratio is about 50:50. What is odd, however, is that the 
positive connotations associated with 奇 (qi or ki) are disappearing and only the negative connotations seem to be emphasized. In English, the term "odd" in "odd number" has meanings such as strange, queer, peculiar, and one-sided. These have mostly negative connotations.

Now, when did the connection between the Japanese and English language in which 奇数 (kisu) became to be referred to as the odd number and 偶数 (gusu), as the even number, occur? Most Japanese mathematical terms were defined by The Physical Society of Japan, which was established in 1877 during the Meiji Era. The definitions in which 奇数 (kisu) became to be referred to as the odd number and 偶数 (gusu), as the even number, were officially established in 1881.

Then, what was the case before the Meiji Era? Were these terms (even number and odd number) translated by the English or the Japanese? Or were there separate original terms for them? For this reason, it is necessary to look into the history of mathematics and the history of numbers in particular.

\section{Odd Numbers Were Regarded as "Good" in Ancient Greece}

The history of numbers begins with natural numbers, evolving to negative numbers, integers, fractions, decimals, rational numbers, irrational numbers, Gauss complex numbers, transcendental numbers, and Kline quaternions. What is discussed here are the natural numbers, namely the ten initial numbers, one to ten.

The aim is to determine when the terms "even number" and "odd number" came to be used. According to Denis Guedj in "L'empire des nombres" (Sogensha Inc.), the Pythagoreans of the fifth and fourth centuries B.C. were the first to distinguish odd numbers from even numbers.

Their use of this concept paved the way for a variety of results. The line of natural numbers is made up of the infinite repetition of even and odd numbers. Since the prime numbers were found in order to classify natural numbers, the classification of even numbers and odd numbers was therefore conducted by the initial prime number "two".

Once the numbers were classified into the two categories, the "odd numbers" and the "even numbers", which set did the ancient Greeks prefer? Wataru Uegaki makes the following point in "The Origin of Greek Mathematics" (NIPPON HYORONSHA CO., LTD.): Pythagoras not only linked everything to numbers, but he tried to express everything in numbers. First, Pythagoras classified numbers into odd numbers and even numbers. Then, since odd numbers 
cannot be divided at will? Aand with the belief that what cannot be broken down is 'complete', he connected odd numbers to "completeness", "mysteriousness", and "finiteness". In contrast, even numbers, because they could be divided into two, were given the opposite attributes.

Aristotle summarized his views on the matter in Vol. 1, Chapter 5 of "Metaphysics" (Iwanami Bunko) as follows. He said there are ten principles, which he listed in a table of oppositions. In other words, there are finite and infinite, odd and even, one and many, right and left, male and female, stillness and motion, straight and crooked, light and darkness, good and evil, squares and rectangles. This list of oppositions is known as the table of categories of the Pythagoras school or the table of contrary concepts. In general, he considered the first-listed concepts to be 'good?f and with 'form' while he considered the second-listed concepts to be 'bad' and 'material'.

\section{Odd Numbers and Even Numbers in the Yin-Yang Thought}

In the times of the ancient Greeks, odd numbers were regarded as 'good?f. This resembles the situation in Yin-Yang thought in China, discussed below. Was Pythagoras of ancient Greece the first to divide numbers into odd and even? I suspected that the ancient Chinese may have been the first. Thus, I looked into the fortunetelling of the Yi Ching, associated with the Yin-Yang thought in China. Yi Ching thought-based fortunetelling originated in the Zhou Dynasty of ancient China, which lasted from the 12th to 3rd century B.C. This is roughly the same time-period as that of ancient Greece. It is not certain which was first; however, there are certain close resemblances between their thought, and therefore, one can presume that there was some sort of reciprocal influence between these two centers of thought.

Now, let me briefly describe Yi Ching fortunetelling, basically, Yin-Yang thought. The core of Yi Ching thought is Yin and Yang (the alternation of masculine and feminine). Originally, there were no 'kanji?f (Chinese characters) for Yin and Yang. Previously, there was 'softness?f and 'hardness?f ('weak?f and 'strong?f). And before that, there were other designations and characters, but I will not delve into that matter here. The Yi Ching thought assigned the principles of movement-stillness and hardness-softness to odd and even numbers. Yang was strong while Yin was compliant. Yang was movement and Yin was stillness. All things of the natural world and the world of humans were arranged under the two Yin-Yang principles. The things that were Yang were the heavens, the sun, fathers, males, benevolence, top, front, brightness, going, 
midday, respect, nobility, and good fortune, while the things that belonged to Yin were the earth, the moon, mothers, females, justice, below, behind, darkness, coming, night, lowliness, humbleness, and misfortune.

Thus, although the Yi Ching thought is based on the concept of two alternating opposites, Yin and Yang, the next point, which is written on page 40 of the Iwanami Bunko edition of the Yi Ching (Book 1), is very important. A male is Yang in relation to a female; however, that same male is Yin in relation to his parents. A female is Yin, but is Yang in relation to her child. Front is Yang in relation to behind, but is Yin in relation to things that are in front of it. Yin and Yang imply infinite change; this is the message imparted by the Yi Ching thought. Hence, Yin and Yang are not universal absolutes. It is emphasized that Yin and Yang are simply polar opposites and not a case of one being superior or inferior to the other. In contrast, it is only in modern times that Yang became to be considered good and superior, and Yin, as bad and inferior.

\section{Contributing to Peace Through an Understanding of Numbers}

In Yin-Yang thought, numbers are divided into yin and yang. The "Transmitted Interpretations of the Zhou-yi" in "Yi-Ching" (Book 2, Iwanami Bunko) arranges the numbers from one to ten in the following, alternating a Heaven and Earth pattern: "Heaven is one, Earth is two; Heaven is three, Earth is four; Heaven is five, Earth is six; Heaven is seven, Earth is eight; Heaven is nine, Earth is ten; Heaven accounts for five numbers and Earth five." The terminology may vary between Heaven and Earth, Yin and Yang, Hard and Soft, and so on, but the numbers are just arranged in a pattern and do not seem to be a conceptual like the odd and even numbering of today.

The emergence of the concept of odd and even really had to wait on the development of mathematics. The following interesting example is found in the "Sunzi San Jing," an ancient Chinese book on arithmetic. There is a pregnant woman who is 29 year old, and the month in which the baby is due is September. The question is whether the baby will be male or female. The answer is male, for the following reason. First of all, the number forty-nine is posited. The month of delivery is added, and the age of the mother is subtracted. Then, one for the Heaven, two for the Earth, three for man, four for the four seasons, five for the five elements, six for the six tones, seven for the seven stars, eight for the winds of the eight directions, and nine for the nine states are subtracted. If the remaining number is an odd number, the baby will be male; if it is even, 
the baby will be female. If one tries this calculation, the answer will not agree. However, it is obvious that the concept of odd and even existed.

The development of the mathematics of ancient Greece, which developed under the influence of Egypt and Babylonia, stopped temporarily, but then led to the development of the mathematics of the Arab world and India. Arabic algebra was transmitted to Europe via Italy in the times of the Crusades. However, the establishment of modern mathematics was still well in the future. Modern mathematics, as represented by Newton, valued rationality and science. Consequently, it seems to have abandoned the ideas from ancient Chinese YinYang thought and ancient Greek philosophies, in which the odd number was male and the even number, female. When counting numbers, odd numbers were incomplete, in-between numbers, while even numbers were certainly the more rational.

In modern times, when the mathematics of the East and that of the West, which developed independently, merged, historical and cultural differences between the terms were probably exposed when the mathematical terminology claimed kisu (奇数) as odd numbers, and gusu (偶数) as even numbers. In contrast to the East, where the odd number was positive and good, in the West, the odd number was incomplete and superfluous.

I have argued that the East has a culture of odd numbers while the West, even numbers. However, what about the Middle East? That would be interesting to look into. Moreover, the sun is positive and good in both the East and the West, whereas in the Middle East, the moon has positive connotations, which is also different from the Yin-Yang meaning in the East. More so, Arabic writing is just the opposite of English; teletext on news broadcast by the Middle Eastern satellite broadcasting station AL Jazeera move from left to right.

Odd numbers and even numbers, the sun and the moon, the direction of writing, and so on are opposing concepts; however, opposites are not eternally opposite. In dialectics, oppositions are contradictions; however, they are unified by sublation and the combination of thesis and antithesis. When discussing the Iraq issue and other international disputes, there are surely matters that cannot be discussed only in political and economic terms. Differences in historical relationships lie deep in individual cultures and histories. I believe that it is possible to find ways leading to peaceful settlements by mutually acknowledging such differences. Although the odd number and even number are mathematical concepts, they seem to be deeply rooted in politics and society as well. (See more Nishiyama, 2004), see [1]. 


\section{References}

[1] Y. Nishiyama, Su no Bunkashi [The Cultural History of Numbers], Keizaishi Kenkyu [Studies in Economic History], 8 (2004), 146-174. 
